\title{
Diagnostic value of transoesophageal echocardiography in suspected haemodynamically significant pulmonary embolism
}

\author{
P Pruszczyk, A Torbicki, A Kuch-Wocial, M Szulc, R Pacho
}

\begin{abstract}
Objective-To assess the value of transoesophageal echocardiography (TOE) for diagnosing suspected haemodynamically significant pulmonary embolism and signs of right ventricular overload at standard echocardiography.

Methods-113 consecutive patients (58 male; 55 female), mean (SD) age 53.6 (13.3) years, in whom there was clinical suspicion of pulmonary embolism and right ventricular overload on transthoracic echocardiography, underwent TOE in addition to routine diagnostic procedures to identify pulmonary artery thrombi.

Results-TOE revealed thrombi in 32 of 51 patients who had suspected acute pulmonary embolism and in 31 of 62 with suspected chronic pulmonary embolism. In one patient a pulmonary angiosarcoma rather than chronic pulmonary embolism was found at surgery. The diagnosis of pulmonary embolism was confirmed in 77 patients by scintigraphy, spiral computed tomography, angiography, or necropsy (reference methods). While TOE failed to provide a diagnosis of pulmonary embolism in 15 of these 77 patients, no false positive findings were reported (sensitivity $80.5 \%$, specificity $97.2 \%$ ). In 11 and 26 cases, respectively, the thrombi were confined to the left or right pulmonary artery. Bilateral thrombi were found in 25 patients. Mobile thrombi were observed only in acute pulmonary embolism (in 19 of 32 patients). No complications of TOE were noted.

Conclusions-TOE permits visualisation of pulmonary arterial thrombi, confirming the diagnosis in the majority of patients with pulmonary embolism and right ventricular overload. This may be useful for prompt decision making in patients with haemodynamic compromise considered for thrombolysis or embolectomy.
\end{abstract}

(Heart 2001;85:628-634)

Keywords: pulmonary embolism; transoesophageal echocardiography

It is generally accepted that patients with acute massive pulmonary embolism resulting in shock or systemic hypotension require thrombolytic treatment. ${ }^{12}$ Moreover, it has been suggested recently that normotensive patients with pulmonary embolism and right ventricular pressure overload found on transthoracic echocardiography may also benefit from such treatment. ${ }^{3-6}$ However, before aggressive treatment is begun reliable confirmation of pulmonary embolism is required. Standard diagnostic methods (pulmonary angiography and lung scintigraphy) are not often performed in patients with suspected pulmonary embolism and right ventricular overload, especially when there is shock or after cardiopulmonary resuscitation. ${ }^{2}$ Such methods also suffer from various limitations in patients with chronic pulmonary embolism. ${ }^{7}$ Therefore there is increasing interest in newer imaging procedures such as spiral computed tomography (CT) and transoesophageal echocardiography (TOE), which are reported to be useful in the diagnosis of both acute and chronic pulmonary embolism. . $^{-11}$

Though various reports have suggested that reliable visualisation of proximal pulmonary artery thromboemboli may be possible by TOE, ${ }^{911}{ }^{12}$ the sensitivity and specificity of this test in the diagnosis of pulmonary embolism has not been assessed systematically.
In this study our aim was to determine prospectively the diagnostic value of TOE in suspected haemodynamically significant pulmonary embolism, defined by the presence of right ventricular pressure overload.

\section{Methods}

We assessed 487 consecutive patients referred to our echocardiographic laboratory on clinical suspicion of acute or chronic pulmonary embolism, using standard transthoracic echocardiography (fig 1). At least three of following five criteria of right ventricular pressure overload were required:

- peak velocity of tricuspid valve insufficiency, corresponding to a right ventricular to right atrial peak systolic gradient of more than $30 \mathrm{~mm} \mathrm{Hg}$;

- enlargement of the right ventricle (more than $27 \mathrm{~mm}$ diameter measured in the parasternal long axis);

- a shortened (<80 ms) pulmonary ejection acceleration time, measured in the right ventricular outflow tract;

- flattening of the intraventricular septum;

- distension of the inferior vena cava (> $20 \mathrm{~mm}$ diameter).

In 338 of the 487 cases there was no evidence of right ventricular pressure overload by these criteria, so those patients were not considered further. A further 36 patients with 


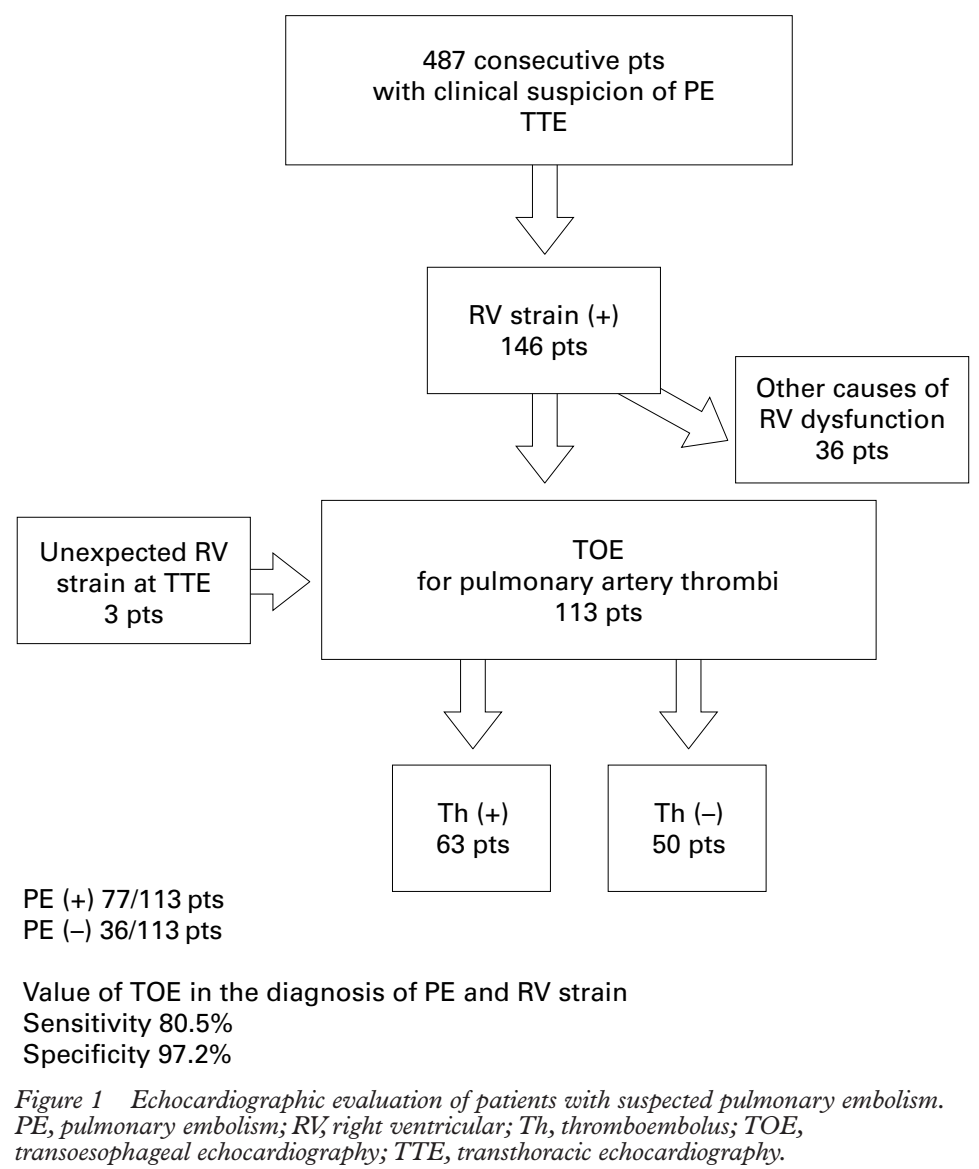

the echocardiographic criteria for inclusion in the study had obvious alternative clinical or echocardiographic causes for right ventricular dysfunction. These included advanced chronic obstructive pulmonary disease in seven patients, and haemodynamically significant valvar heart disease or left ventricular dysfunction in another 29. These subjects were also excluded from the study.

The remaining patients, who had signs of right ventricular strain on transthoracic echocardiography and no alternative explanation for this on clinical and echocardiographic grounds, formed the study group with suspected haemodynamically important pulmonary embolism. These were then investigated by TOE. Three further patients, who were referred to the echocardiographic laboratory without clinical suspicion of pulmonary embolism but with unexpected and unexplained right ventricular pressure overload on transthoracic echocardiography, were also included in the TOE study group. The group with unexplained right ventricular pressure overload finally comprised 113 consecutive patients (58 men and 55 women), mean (SD) age, 53.6 (16.3) years. Of these 113 patients, 51 who were examined within 14 days after the onset of clinical symptoms were suspected of having acute pulmonary embolism. Their mean duration of symptoms was 5.0 (3.5) days. The other 62 patients in the study group, who had a longer history of disease (80.7 (85.6) days; range 30 days to 14 months), were suspected of having chronic pulmonary embolism.
TRANSOESOPHAGEAL ECHOCARDIOGRAPHY

The first 27 consecutive patients were investigated with a single plane transoesophageal 5.0 MHZ transducer and a SONOS 500 system (Hewlett-Packard, Palo Alto, California, USA), and the remaining 86 subjects with a biplane 5.0 MHZ transoesophageal probe and a SONOS 1000 system.

The echocardiographic laboratory was supplied with standard resuscitation equipment. Oxygen was delivered through nasal prongs at 2-4 1/min 10 minutes before insertion of the transducer. An intravenous line was introduced into a right antecubital vein. Blood pressure was measured with a mercury sphygmomanometer just before and after the examination. During the examination the patients were monitored continuously using transcutaneous pulse oximetry and electrocardiography. The patient was placed on the left side and after superficial anaesthesia of the pharynx with $2 \%$ lignocaine (lidocaine) spray the transducer was introduced into the middle segment of the oesophagus in the standard way. No sedation was used.

The examination begun with an evaluation of the main pulmonary artery, after which the right pulmonary artery was investigated in both the transverse and the longitudinal planes up to the initial parts of lobar arteries (intermediate trunk and right upper lobe artery). After the examination of the right pulmonary artery the transducer in the transverse plane was rotated counterclockwise in order to evaluate the left pulmonary artery. Here the echocardiographic continuity of the artery was often lost owing to the interposition of the left main bronchus, which shielded the middle segment of the left main pulmonary artery from the ultrasound beam. However, the distal part of this vessel was identified by its position with respect to the descending aorta, by the characteristic branching of the left upper lobe artery, and by the appearance of profuse air microbubbles following saline injection into the right antecubital vein. The pulmonary arteries were evaluated in the transverse and the sagittal planes whenever possible. Efforts were made to visualise the vessels as distally as possible, including the initial parts of the lobar arteries on both sides.

Morphological evaluation of the pulmonary arteries was followed by colour and spectral Doppler analysis for the assessment of possible flow disturbances caused by intraluminal masses. Care was taken to exclude aortic dissection as a potential cause of nonthrombotic obstruction of the proximal pulmonary arteries from mechanical compression.

The study was completed by evaluating the right atrium, looking for thrombi and intraatrial septal defects, especially of the sinus venosus type. All four pulmonary veins were identified in order to assess potential asymmetry of blood flow, and to rule out partial anomalous venous return as a cause of right ventricular overload. 
Evaluation of transoesophageal echocardiography Intraluminal masses visualised by TOE in the pulmonary arteries were identified by an experienced echocardiographer as thromboemboli when they had distinct borders and a different echo density from blood and vascular wall. Detection of blood flow disturbances caused by intraluminal masses and motion of intraarterial structures that differed from the surrounding tissue were helpful in the diagnosis of thrombi. The echocardiographic diagnosis of pulmonary artery thromboemboli was always made with the understanding that it could serve as sufficient justification for potential thrombolysis or surgery. Thus for intrapulmonary masses to be reported as thrombi they had to be unequivocally visualised during TOE.

All examinations were recorded on videotape for off-line blinded re-evaluation by another experienced echocardiographer. Sixty five patients underwent transthoracic echocardiography followed by TOE as the first diagnostic procedures in the work up of suspected pulmonary embolism. Although in the remaining 48 patients lung scintigraphy, spiral CT or pulmonary angiography preceded TOE, the echocardiographic team was not informed of the results of those investigations.

DIAGNOSIS OF PULMONARY EMBOLISM

All the study patients underwent diagnostic procedures to confirm or exclude pulmonary embolism. Lung scintigraphy was performed in every case and was evaluated according to the PIOPED (prospective investigation of pulmonary embolism) criteria. $^{13}$ Results indicating pulmonary embolism with high probability were regarded as sufficient to confirm pulmonary embolism, while normal or near normal perfusion excluded pulmonary embolism.

Seventy four patients were examined by spiral CT, which was performed and assessed according to a previously described protocol. ${ }^{14}$ Detection of thromboemboli at least in segmental arteries confirmed pulmonary embolism, whereas negative result did not exclude it.

In cases with non-diagnostic scintigraphy and negative spiral CT, pulmonary angiography was performed..$^{15}$ In patients scheduled for surgery, all imaging modes were used. Altogether angiography was done in 24 cases.

Pulmonary embolism was also confirmed by typical findings at surgery (12 cases) or necropsy (four patients).

The protocol of this study was approved by our institutional ethics committee. All patients gave informed consent.

\section{Results}

DIAGNOSTIC VALUE OF TRANSOESOPHAGEAL ECHOCARDIOGRAPHY

Transoesophageal echocardiography revealed unequivocal masses in the pulmonary arteries in 63 of 113 patients with suspected severe pulmonary embolism. Although pulmonary embolism was eventually confirmed by standard diagnostic tests in all these patients, TOE provided one false positive diagnosis of chronic pulmonary embolism in a patient whose
Table 1 Diagnostic value of transoesophageal echocardiography in the diagnosis of haemodynamically significant pulmonary embolism

\begin{tabular}{lccl}
\hline & Total PE & Acute PE & Chronic PE \\
\hline Suspected (n) & 113 & 51 & 62 \\
PE (+) (n) & 77 & 42 & 35 \\
PE (-) (n) & 36 & 9 & 27 \\
TOE (+) (n) & 63 & 32 & 31 \\
TOE $(-)(\mathrm{n})$ & 50 & 19 & 31 \\
TOEf $(-)(\mathrm{n})$ & 15 & 10 & 5 \\
TOEf $(+)(\mathrm{n})$ & $1^{\star}$ & 0 & $1^{\star}$ \\
Sensitivity (\%) & 80.5 & 76.1 & 85.7 \\
Specificity (\%) & 97.2 & 100 & 96.4 \\
\hline
\end{tabular}

${ }^{\star}$ One patient with leiomyosarcoma of pulmonary artery PE, pulmonary embolism; PE (+), pulmonary embolism confirmed by routine methods; PE $(-)$, pulmonary embolism excluded by routine methods; TOE $(+)$, positive diagnosis of pulmonary embolism at transoesophageal echocardiography; TOE (-), negative diagnosis of pulmonary embolism at transoesophageal echocardiography; TOEf $(-)$, false negative transoesophageal echocardiography; TOEf $(+)$, false positive transoesophageal echocardiography.

intrapulmonary mass was identified as a leiomyosarcoma following successful surgical removal of the structure. Interestingly, both angiography and spiral CT also yielded a false positive diagnosis of chronic pulmonary embolism. On the other hand pulmonary embolism was diagnosed in 15 patients in whom TOE failed to detect thrombi (table 1). In nine of these, spiral CT or angiography detected thrombotic obstruction distal to the lobar branches of the pulmonary arteries, while scintigraphy showed segmental defects. In six others centrally located thrombi were missed during TOE. Altogether pulmonary embolism was diagnosed in 77 patients - by high probability lung scintigraphy in 49 cases, spiral CT in 32, angiography in 24, and necropsy in four.

In the remaining 35 patients in whom pulmonary embolism was eventually excluded, right ventricular pressure overload was ultimately attributed to primary pulmonary hypertension in 12, parenchymal lung diseases in 14, and congenital heart disease in nine (sinus venosus atrial septal defect $(n=7)$, ostium secundum atrial septal defect $(n=1)$, and Ebstein's anomaly $(n=1)$.

Altogether, the sensitivity of TOE in diagnosing severe pulmonary embolism reached $80.5 \%$, with a specificity of $97.2 \%$ (table 1 ).

Localisation of pulmonary artery thromboemboli Intraluminal masses were echocardiographically visualised in 63 cases. In one patient with bilateral masses extending from the pulmonary trunk to the proximal parts of lobar arteries on both sides a leiomyosarcoma was diagnosed at surgery. Thus thomboemboli were found on TOE in 62 patients with proven pulmonary embolism. In 25 of these, thrombi were found bilaterally, while in the remaining 37 they were limited to one side. In 26 cases masses were found in the right pulmonary artery or in its branches only, whereas in the 11 remaining patients only left sided thromboemboli were visualised (fig 2). Thromboemboli were found in the right main or left main pulmonary arteries in 56 patients, and they were usually detected in the region of branching to lobar arteries of the right or left main pulmonary 


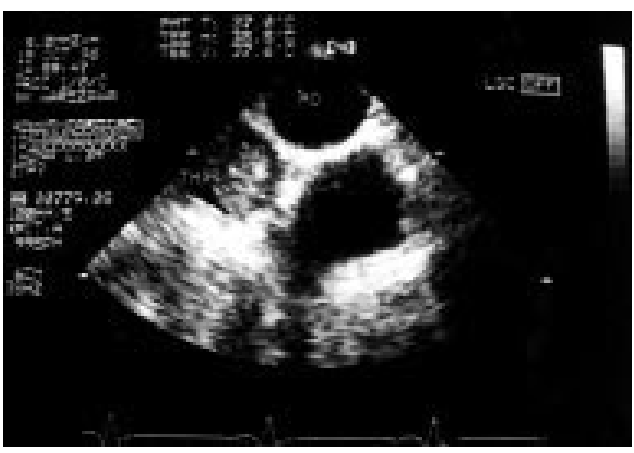

Figure 2 The distal part of the left pulmonary artery almost totally occluded by thrombus (TH) in a patient with acute pulmonary embolism. The left pulmonary artery is closely related to the descending aorta $(A O)$. ART, artefact of aorta.

Table 2 Localisation of thromboemboli in main and/or lobar pulmonary arteries detected at transoesophageal echocardiography in 62 of 77 patients with haemodynamically significant pulmonary embolism

\begin{tabular}{ll}
\hline & Thromboemboli \\
\hline Only main pulmonary arteries & $50.0(31)$ \\
Main and lobar pulmonary arteries & $40.3(25)$ \\
Only lobar arteries & $9.7(6)$ \\
\hline
\end{tabular}

Values are \% (n).

artery (33 patients and 30 patients, respectively). However, evaluation the proximal parts of the lobar arteries by TOE made it possible to diagnose pulmonary embolism in an additional six patients in whom thrombi were limited to these vessels (four with thrombi in the intermediate artery, one with thrombus in the right upper lobe artery, and one with thrombus in the left upper lobe artery) (table 2).

\section{Morphology of the thromboemboli}

The dimensions, morphology, mobility, and echodensity of the thromboemboli were assessed. The dimensions of the thrombi varied from $7 \times 7 \mathrm{~mm}$ up to $60 \times 14 \mathrm{~mm}$, with a mean (SD) value of $24.7(9.2) \mathrm{mm} \times 13.4(1.6) \mathrm{mm}$. Small thrombi were usually lodged in the proximal parts of the lobar arteries. In addition, in two cases of pulmonary embolism total occlusion of one main pulmonary artery was diagnosed: in one of these, who had acute pulmonary embolism, the left pulmonary artery was occluded, while in the other, who had chronic pulmonary embolism, thromboemboli occluded the right pulmonary artery.

Mobile, usually "snake-like", thrombi were visualised in 19 of 32 patients with acute pulmonary embolism $(59.4 \%)$. In the remaining 13 patients $(40.6 \%)$ only immobile, irregular thrombi were found. Immobile thrombi were detected in all 30 cases with chronic pulmonary embolism (fig 3). Interestingly, in 20 of these $(66.6 \%)$ thromboemboli were perimural, closely adhering to the vascular wall. Moreover, in seven patients with chronic pulmonary embolism an increased echodensity of thrombotic material was present.

TRANSOESOPHAGEAL ECHOCARDIOGRAPHY AT FOLLOW UP

Sixteen patients with acute pulmonary embolism underwent TOE after four weeks of treatment (three with thrombolytic drugs and 13

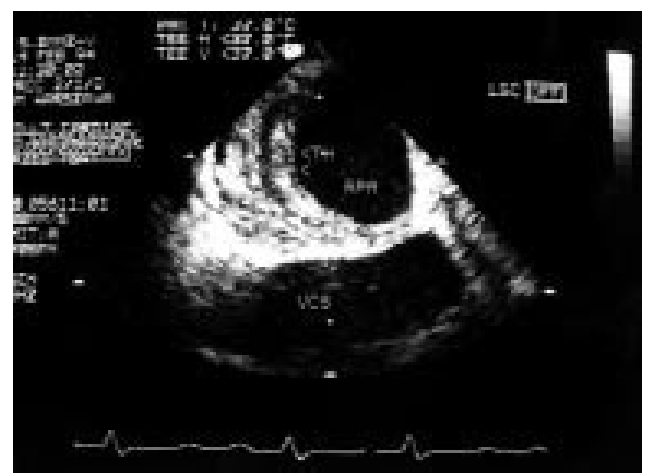

Figure 3 Perimural immobile thrombus (TH) of the right pulmonary artery (RPA) in a patient with chronic pulmonary embolism. VCS, superior vena cava.

with anticoagulants). The thrombi had completely resolved in 13 patients, while intraluminal masses were still present in the remaining three, though they were much smaller. In the latter, repeated TOE after a further five months of oral anticoagulation showed total resolution of the thrombi. No clear relation was observed between the mobility and morphology of the thrombotic material and the rate of thrombus resolution in the patients with acute pulmonary embolism; in all these 16 patients right ventricular function recovered completely.

Transoesophageal echocardiography was also repeated after four weeks of oral anticoagulation in 13 patients with chronic pulmonary embolism scheduled for surgery. In all these cases the thromboembolic masses remained unchanged at one month of follow up. After another five months of anticoagulant treatment eight patients underwent a third TOE; in two of these only minor changes in the morphology of the thromboemboli were found, while in the other six the intraluminal masses were unchanged. Despite continuous oral anticoagulation no significant decrease in the right ventricular pressure overload was observed at follow up in these patients. Altogether, 12 patients with chronic pulmonary embolism underwent thrombendarterectomy. Intraluminal thrombus was removed in 11 of these cases, and one was found to have a leiomyosarcoma, as stated above. Control TOE performed after surgery revealed no intrapulmonary masses.

ADDITIONAL INFORMATION

In 59 haemodynamically stable patients with suspected pulmonary embolism who tolerated transoesophageal echocardiography well, flow disturbances caused by intrapulmonary masses were assessed by colour and spectral Doppler. In 46 of these pulmonary embolism was ultimately diagnosed. Haemodynamically significant intrapulmonary pressure gradients varying from $20-31 \mathrm{~mm} \mathrm{Hg}$ (mean, 23.5 (5.1) $\mathrm{mm} \mathrm{Hg}$ ) caused by intraluminal masses were found in four patients (two with chronic and two with acute pulmonary embolism). In all these cases, intrapulmonary pressure gradients were recorded in the lobar arteries.

To exclude anomalous pulmonary venous return as a potential cause of right ventricular overload, the morphology of the pulmonary 
veins and the blood flow were assessed qualitatively. The pulmonary veins were investigated in 82 patients, while in the remaining $31-$ mostly with massive pulmonary embolismthe examination was not extended beyond the time necessary for unequivocal visualisation of pulmonary artery thrombi. Despite the normal structure of the pulmonary veins in all these patients, significant asymmetry of blood flow in these vessels was detected in seven. Interestingly, in all cases lung scintigraphy confirmed pulmonary embolism and revealed pronounced defects on pulmonary perfusion corresponding to the regions of decreased blood return from the pulmonary veins.

In 88 patients with right ventricular pressure overload and in whom an atrial septal defect was excluded, the intra-atrial septum was investigated for a patent foramen ovale. This was detected in 30 patients $(34.1 \%)$. Although it seemed to be more common in patients with chronic pulmonary embolism (13/33 (39.4\%)) than in those with acute pulmonary embolism or those in whom pulmonary embolism was excluded (12/38 (31.5\%) and 5/17 (29.4\%), respectively), the difference was not significant. While paradoxical embolism was not systematically assessed, it is worth mentioning that two patients with acute ischaemic strokes were initially referred to the echocardiographic laboratory because of a suspicion of cardiogenic embolism. In both these cases thrombi were found in the pulmonary arteries in conjunction with a patent foramen ovale. In another patient with a patent foramen ovale, an acute embolic ischaemic stroke occurred six months after the initial diagnosis of pulmonary embolism.

DURATION AND SAFETY OF TRANSOESOPHAGEAL ECHOCARDIOGRAPHY

The mean duration of TOE in patients with suspected pulmonary embolism was $9.6 \mathrm{~min}$ utes, varying from 1.2-18 minutes. In patients with poor haemodynamic status or intolerance of the transoesophageal probe the examination was limited to the evaluation of the pulmonary arteries. During the shortest examination, which lasted 72 seconds, massive bilateral thrombi were visualised. In three cases the examination was terminated prematurely, because of bradycardia in one case and because of increasing hypoxaemia $\left(\mathrm{SO}_{2}<70 \%\right)$ despite continuous oxygen delivery in two others. However, in the study cohort as a whole, with all patients receiving oxygen during TOE, no significant increase in hypoxaemia was found. At the time of probe insertion and at the end of the examination, heart rate did not differ significantly (94.6 (3.6) and 93.6 (5.7) beats/ min, respectively). Although $18.6 \%$ of the patients investigated experienced syncope within three days before TOE, at the time of the examination all the patients were haemodynamically relatively stable, with no need for catecholamine infusion or assisted ventilation. Before insertion of the probe systolic blood pressure was below $100 \mathrm{~mm} \mathrm{Hg}$ in 32 patients, with a mean value of 121.9 (19.9) $\mathrm{mm} \mathrm{Hg}$ in the group as a whole. In no patient did TOE result in significant hypotonia or cardiac arrhythmia, and no other important complications occurred.

\section{Discussion}

Pulmonary embolism is still one of the major causes of in-hospital mortality. ${ }^{16}{ }^{17}$ Prompt and accurate diagnosis is especially important in haemodynamically unstable patients, because they may require urgent thrombolysis or surgery. The MAPPET (management strategy and prognosis of pulmonary embolism) registry showed that in the most compromised patients with suspected acute pulmonary embolism routine diagnostic procedures such as angiography or lung scintigraphy are performed infrequently, and the diagnosis of pulmonary embolism is based mainly on clinical evaluation and the presence of right ventricular overload on transthoracic echocardiography. ${ }^{2}$ However, right ventricular strain is only an indirect sign of pulmonary embolism and may be present in other cardiopulmonary conditions such as acute respiratory distress syndrome. ${ }^{18} 19$ Inaccurate diagnosis may lead to unnecessary thrombolysis with all its risks. As it has been reported that almost one third of patients with acute pulmonary embolism who receive thrombolysis have relative contraindications to such treatment, definitive confirmation of pulmonary embolism is especially important in this group of patients. ${ }^{2}$ A bedside method of promptly confirming pulmonary embolism would be very useful, as would a method for making a differential diagnosis of acute pulmonary embolism in cases of decompensated chronic thromboembolic pulmonary hypertension.

In patients with chronic pulmonary embolism the time factor is not so important and elective pulmonary angiography can usually be arranged. However, pulmonary angiography may not be a very precise way of evaluating proximal perimural thrombi. ${ }^{7}$ Detection of thromboemboli in the central arteries is an essential feature of the assessment of patients for surgical treatment. ${ }^{20}$

In the present study the value of transoesophageal echocardiography was assessed prospectively in the diagnosis of suspected haemodynamically significant acute and chronic pulmonary embolism, defined by the presence of right ventricular overload on transthoracic echocardiography. Intrapulmonary masses were detected at TOE in over $50 \%$ of 113 consecutive patients. In 62 of these, routine diagnostic methods confirmed pulmonary embolism, whereas in only one case with suspected chronic pulmonary embolism was a false positive diagnosis made, using all imaging methods including TOE. In this case, the intraluminal mass was found to be a sarcoma of the pulmonary arteries and was diagnosed at surgery. Problems with the preoperative identification of pulmonary sarcomas have been described before. ${ }^{21}$

Our overall results with TOE in patients with evidence of right ventricular pressure overload showed a fair sensitivity of $80.5 \%$ and a high specificity of $97.2 \%$. High specificity of TOE 
was achieved because only unequivocal intrapulmonary masses with distinct borders and different echodensity from the vascular walls were reported as thromboemboli. The diagnosis of thrombi was always made on the basis that the results of TOE could be sufficient justification for thrombolysis or embolectomy. Because of topographic limitations TOE is unable to visualise the distal pulmonary arteries. Thus TOE cannot exclude pulmonary embolism, though in our experience it can provide prompt, reliable confirmation of pulmonary embolism, fully justifying the decision to proceed with thrombolysis or surgery. In earlier reports, the sensitivity of TOE for diagnosing pulmonary embolism suffered from an inadequate assessment of the left pulmonary artery. ${ }^{11} 1222$ Indeed, in the majority of cases described in those studies the middle segment of the left pulmonary artery was shielded by the left main bronchus. This part of the vessel was thus impossible to visualise, and continuity was usually lost. In our study, however, rotation of the probe allowed the distal part of the left pulmonary artery, with the characteristic branching of the left upper lobe artery, to be visualised close to the descending aorta in $80 \%$ of the subjects, even in those without pulmonary hypertension. If we had limited the transoesophageal examination to the main and right pulmonary arteries, TOE would have identified thrombi in 51 cases of pulmonary embolism. Evaluation of the left pulmonary artery allowed us to detect thrombi in an additional 11 patients with embolic material limited to the left side. TOE confined to an evaluation of the main and the right pulmonary arteries achieved a sensitivity of $66.2 \%$, similar to that reported by other investigators, ${ }^{11}{ }^{12} 22$ but our modification of the technique of assessing the distal left pulmonary artery increased the sensitivity to $80.5 \%$. It should also be emphasised that by extending TOE to the proximal parts of the lobar arteries we were able to diagnose thrombi in an additional six patients $(7.8 \%)$.

Mobile thrombi seem to be characteristic of acute pulmonary embolism, although immobile masses were detected in both acute and chronic pulmonary embolism. Increased echodensity of the thromboemboli was a feature of chronic pulmonary embolism. Whether the presence of mobile thrombi can predict outcome, or should be taken into account when making decisions about treatment, remains to be established.

Apart from the direct visualisation of thrombotic material in the pulmonary arteries, TOE may provide additional data that could be useful in the diagnosis of pulmonary embolism. For example, the intrapulmonary pressure gradient was recorded in four of the patients investigated. This confirms the haemodynamic significance of visualised thrombus and may be useful in differentiating between pulmonary embolism and local thrombosis in the proximal parts of the pulmonary arteries, which is described in patients with prolonged severe pulmonary hypertension..$^{23}$ Another potentially helpful observation is the pronounced asymmetry of venous blood inflow into the left atrium which was observed in seven patients, despite normal morphology of the pulmonary veins. In all of these, a decrease in blood return corresponded to the region of significantly reduced perfusion observed at lung scintigraphy.

CLINICAL IMPLICATIONS OF THE STUDY

Transoesophageal echocardiographic examination of the central pulmonary arteries seems to be a reliable and safe method of definitively confirming haemodynamically significant pulmonary embolism, though it is not capable of excluding their presence. As a prompt bedside examination, TOE can rapidly detect clots in $80 \%$ of cases of pulmonary embolism presenting with right ventricular overload. We feel that in selected cases, and considering the possibility of bedside evaluation, TOE may be the method of first choice even if spiral CT is available. ${ }^{14}$ TOE may be the method of first choice in haemodynamically compromised patients who require urgent thrombolytic treatment or surgery.

It has been reported that the diagnosis of pulmonary embolism can be established by TOE even during resuscitation. ${ }^{24}$ Direct visualisation of intrapulmonary thrombi may be of particular importance in patients with suspected massive pulmonary embolism and a coexisting relative contraindication for thrombolysis. TOE had been used by several investigators in the diagnostic work up of patients with suspected severe pulmonary embolism. ${ }^{25}$

The role of TOE in suspected chronic pulmonary embolism consists mainly of evaluating the proximal extension of thrombi, which may be useful before surgical treatment is undertaken. However, it should be born in mind that TOE may be able to identify other causes of severe chronic pulmonary hypertension such as a sinus venosus type defect of the intra-atrial septum.

\section{LIMITATIONS OF THE STUDY}

Our results apply only to patients with right ventricular pressure overload and without known pre-existing haemodynamically significant cardiopulmonary disease. Thus our results cannot be extrapolated to an unselected group of patient with suspected pulmonary embolism. The sensitivity of TOE would most probably be much lower in such a setting. Moreover, there is a learning curve for evaluating the pulmonary arteries at TOE. Thus the personal experience of the echocardiographic team is likely to affect the value of the method in diagnosing pulmonary embolism. The results of our study were obtained in a centre specially interested in this application of TOE, and this may be relevant in explaining why other investigators have reported a lower sensitivity and specificity for the method. ${ }^{11}{ }^{12} 22$

The patients who were studied underwent various imaging examinations to confirm or exclude pulmonary embolism. The absence of a standard diagnostic protocol can be regarded as a limitation of our study. A high probability of pulmonary embolism on the one hand, and normal or near normal lung scintigraphy on the 
other, were considered sufficient to confirm or exclude pulmonary embolism, respectively. Pulmonary angiography was performed in the minority of patients.

A relatively high sensitivity and specificity were found despite the fact that the first 27 patients were investigated with a monoplane probe. The use of an omniplane probe in the whole group might have slightly improved the visualisation of the pulmonary arteries and resulted in more frequent detection of thrombi in the lobar vessels.

\section{CONCLUSIONS}

TOE is a reliable and safe method for promptly confirming suspected pulmonary embolism in patients with right ventricular overload on transthoracic echocardiography. Although evaluation of both main pulmonary arteries at TOE can detect thromboemboli in the majority of patients with haemodynamically significant pulmonary embolism, extension of the examination to the initial parts of the lobar arteries may further increase the diagnostic value.

This study was partially supported by grant KBN 1398/P05/ 2000/19.

1 Jerjes-Sanches C, Ramirez-Rivera A, Garcie M, et al. Streptokinase and heparin versus heparin alone on massive pulmonary embolism: a randomized controlled trial. $\mathcal{F}$ Thromb Thrombolysis 1995;2:227-9.

2 Kasper W, Konstantinides S, Geibel A, et al. Management strategies and determinants of outcome in acute major pulmonary embolism: results of a multicenter register. $\mathcal{f} A m$ Coll Cardiol 1997;30:1165-71.

3 Goldhaber SZ, Haire WD, Feldstein ML, et al. Alteplase versus heparin in acute pulmonary embolism: randomised trial assessing right-ventricular function and pulmonary perfusion. Lancet 1993;341:507-11

4 Kasper W, Konstantinides S, Geibel A, et al. Prognostic significance of right ventricular afterload stress detected by echocardiography in patients with clinically suspected pulmonary embolism. Heart 1997;77:346-9.

5 Ribeiro A, Lindmarker P, Juhlin-Dannfelt A, et al. Echocardiography Doppler in pulmonary embolism: right Echocardiography Doppler in pulmonary embolism: right ventricular dysfunction as
Heart $\mathcal{7} 1997 ; \mathbf{1 3 4}: 479-87$

6 Goldhaber SZ, Visani L, De Rosa M. Acute pulmonary embolism: clinical outcomes in the international cooperative pulmonary embolism registry (ICOPER). Lancet 1999;353:1386-9.
7 Brown KT, Bach AM. Paucity of angiographic findings despite extensive organized thrombus in chronic thromboembolic pulmonary hypertension. I Vasc Intervent Radiol 1992;3:99-102.

8 Gefter WB, Hatabu H, Holland GA, et al. Pulmonary embolism: recent developments in diagnosis with CT and MRI imaging. Radiology 1995;197:561-74.

9 Nixdorff U, Erbel R, Drexler $M$, et al. Detection of thromboembolus of the right pulmonary artery by transesophageal two-dimensional echocardiography. $A m \mathcal{F}$ Cardiol 1988;61:488-9.

10 Remy Jardin M, Remy J, Deschildre F, et al. Diagnosis of pulmonary embolism with spiral CT: comparison with pulmonary angiography and scintigraphy. Radiology 1996; 200:699-706.

11 Wittlich N, Erbel R, Eichler A, et al. Detection of central pulmonary artery thromboemboli by transesophageal pulmonary artery thromboemboli by transesophageal embolism. F Am Soc Echocardiogr 1992;5:515-24.

12 Fournier P, Augusseau Richard MP, Charbonnier B, et al. Contribution of transesophageal echocardiography to the diagnosis of pulmonary embolism. Arch Mal Coeur Vaiss 1994;87:459-65.

13 PIOPED investigators. Value of ventilation/perfusion scan in acute pulmonary embolism: results of prospective investigation of pulmonary embolism (PIOPED). $7 A M A$ 1990; 263:2753-96.

14 Pruszczyk P, Torbicki A, Pacho R, et al. Noninvasive diagnosis of suspected severe pulmonary embolism: transesophageal echocardiography vs spiral CT. Chest $1997 ; 112: 722-8$.

15 Greenspan RH. Angiography in pulmonary embolism. In: Abrams HL, ed. Angiography: vascular medicine and interventional radiology. Boston; Little Brown, 1983:803-16.

16 Dalen JE, Alpert JS. Natural history of pulmonary embolism. Prog Cardiovasc Dis 1975;17:259-70.

17 Karwinski B, Svendsen E. Comparison of clinical and postmortem diagnosis of pulmonary embolism. I Clin Pathol $1989 ; 42: 135-9$

18 Jardin F, Dubourg O, Bourdarias JP. Echocardiographic pattern of acute cor pulmonale. Chest 1997;111:209-17.

19 McConnell MV, Solomon SD, Rayan ME, et al. Regional right ventricular dysfunction detected by echocardiography in acute pulmonary embolism. Am 7 Cardiol 1996;78:46973.

20 Moser KM, Auger WR, Fedullo PF, et al. Chronic thromboembolic pulmonary hypertension: clinical picture and surgical treatment. Eur Respir f 1992;5:334-42.

21 Parish J, Rosenow EC, Swensen SJ, et al. Pulmonary artery sarcoma. Chest 1996;110:1480-8.

22 Antakly-Hanon Y, Vieillard-Baron A, Qanadli SD, et al. The value of transesophageal echocardiography for the diagnosis of pulmonary embolism with acute pulmonary heart disease. Arch Mal Coeur Vaiss 1998;91:843-8.

23 Moser KM, Fedullo PF, Finkbeiner WE, et al. Do patients with primary pulmonary hypertension develop extensive central thrombi? Circulation 1995;91:741-5.

24 van der Wouw PA, Koster RW, Delemarre BJ, et al. Diagnostic accuracy of transesophageal echocardiography during cardiopulmonary resuscitation. F Am Coll Cardiol 1997;30: cardiop

25 Rosenow EC. Venous and pulmonary thromboembolism: an algorithmic approach to diagnosis and management. Mayo Clin Proc 1995;70:45-9. 